\title{
The use of circular fine wire frames for the treatment of displaced intra-articular calcaneal fractures
}

\author{
C. Mauffrey $\cdot$ P. Klutts $\cdot$ D. Seligson
}

Received: 24 July 2008/Accepted: 10 November 2008/Published online: 12 December 2008

(C) Springer-Verlag 2008

\begin{abstract}
Background The treatment of displaced intra-articular calcaneal fractures is still controversial.

Materials and methods Sixteen consecutive patients admitted at University Hospital with intra-articular fractures were treated with fine wire circular frames and followed up at an average of 160 days from their injuries. We focused on radiological outcome and functional outcome using a patient-based questionnaire.

Results We had no secondary reconstruction procedures. With the numbers available, the difference between the preoperative values and the follow-up measurements for Böhler's angle, Gissane's angle and posterior subtalar joint space was not statistically significant $(P=0.8, P=0.2$, and $P=0.4$, respectively). The standardized AAOS FAS ranged from 42 to 96 , with a mean of 80 and a standard deviation of 19.

Conclusions Fine wire circular frame is a good alternative to ORIF in displaced intra-articular calcaneal fractures, yielding good patient function, a high return-to-work rate and a low complication rate.
\end{abstract}

\footnotetext{
C. Mauffrey · P. Klutts · D. Seligson

University of Louisville Hospital, Louisville, KY, USA

P. Klutts

e-mail:dpm2007@yahoo.com

D. Seligson

e-mail: seligson@louisville.edu

C. Mauffrey $(\square)$

456 Warwick Road, B911AG Solihull, UK

e-mail: cmauffrey@yahoo.com
}

Keywords Calcaneal fracture $\cdot$ Small wire circular frame $\cdot$ Functional outcome

\section{Introduction}

The calcaneus is the most commonly fractured bone in the foot, representing $60 \%$ of the fractures occurring in the tarsal bones [16]. The treatment of this injury is still controversial, especially for displaced intra-articular fractures.

Possible treatments for calcaneal fractures include elevation and application of plaster cast with early range of movement, closed manipulation and pin reduction with application of plaster, and open reduction internal fixation. This latter form of treatment is becoming increasingly popular. The goal of the treatment of intra-articular displaced fractures is to restore the articular surface, shape, length and height of the calcaneus to optimize the functional outcome. There is no long-term evidence that this is achieved more favorably with open reduction and internal fixation than with other forms of treatment. Furthermore, the morbidity linked with ORIF of the calcaneus is not negligible (10-25\%) [4].

In addition, patients with significant soft tissue swelling or open wounds are not ideal candidates for extensive incisions. Fine wire frame external fixators are being used for the treatment of displaced intra-articular fractures of the calcaneus with soft tissue compromise, or in patients with moderately displaced fractures.

There is still controversy over whether displaced intraarticular calcaneal fractures should be treated operatively or nonoperatively. The Canadian study from 2001 [7] showed that without stratification of the groups, the functional results obtained after nonoperative care of displaced intra-articular calcaneal fractures were equivalent to those 
obtained after operative care. However, after unmasking the data by removing the patients who were receiving Workers Compensation, the outcomes were significantly better in some groups of surgically treated patients.

We hypothesize that the most complex intra-articular calcaneal fractures go on to subtalar fusion, and that the heel can be shaped as well with an external fixator at less risk. We present a consecutive series of patients with displaced intra-articular calcaneal fractures treated between January 2004 and 2007 using a fine wire circular frame.

\section{Materials and methods}

We reviewed a consecutive series of 16 patients admitted with a diagnosis of displaced intra-articular calcaneal fracture between January 2004 and 2007. All of the patients gave informed consent for inclusion in the study, and the study was authorized by the local ethical committee and performed in accordance with the ethical standards of the 1964 Declaration of Helsinki. All patients were operated on at our institution by one surgeon (DS). The case notes and imaging studies were reviewed. All patients were treated with fine wire frame external fixators and were followed up at our clinic. Sex, premorbid state, social history, mechanism of injury and other associated injuries were noted. We examined radiological features of the fracture preoperatively and at their most recent outpatient follow-up (Böhler's angle [1], Gissane's angle [6] and posterior subtalar joint space) to assess the quality of radiological reduction; two independent observers performed this examination. Preoperative investigations included plain lateral radiographs, axial radiographs and anteroposterior and oblique views to assess anterior process and calcaneocuboid joint. A computed tomography (CT) scan was also obtained. These investigations enabled us to plan the direction of distraction and deformity correction.

We followed patients' progress in the clinic, looking at complications, return to work status and total time in frame, and we assessed patients function using a patientbased functional outcome score, the American Academy foot and ankle score (AAFAS) [8], which we performed via telephone at a minimum of one year from the injury (12-48 months).

We used the Essex Lopresti classification [3]. Essex Lopresti subdivided these fractures into two distinct subtypes. The tongue type occurs when the secondary fracture line extends directly posteriorly, producing a large superior, posterior and lateral fragment, with the rest of the body forming the inferior fragment. The joint-depression type, which is the more frequent of the two, occurs when the secondary fracture line begins at the crucial angle, extends posteriorly, but deviates dorsally, to exit the bone just posterior to the posterior articular facet. This fragment contains most of the posterior facet.

Amongst the 16 patients, five were tongue type and 11 were joint depression types. There were four females and 12 males. Two of the calcaneal fractures were open. Nine of these fractures were isolated injuries, whilst the remaining seven patients had other fractures associated with the calcaneal injury, including one tibial plateau fracture, one pelvic fracture, two patients with other foot fractures, one patient with bilateral patella fractures, and one patient with a pilon fracture associated with a pelvic and acetabular fracture, which were both treated nonoperatively.

The mechanism of injury was one gunshot wound, six motor vehicle accidents and nine falls from height. Five fractures were Essex-Lopresti tongue-type injuries, while the remaining 11 injuries were joint-depression type.

The average time from injury to surgery was nine days $(0-42)$, with an average time spent in the frame of 54 days (26-95). All patients were followed up in our clinic until an average follow-up of 160 days (15-295) from their injury.

Statistical analysis of functional and radiological outcome was performed using Student's $t$-test for two samples, assuming unequal variances. All other means were tested by Student's $t$-test for paired samples. The results were considered significant if $P$ was less than 0.05 in a two-tailed study.

Assessment of soft tissue is key prior to undertaking any form of surgery. $60-70 \%$ of calcaneal fractures are associated with a grade I or II soft tissue injury [12].

All patients had plain lateral, axial, anteroposterior and oblique radiographs taken. Contralateral X-rays are especially important, because they help to establish the overall architecture of the calcaneus, in particular the overall height, Böhler and Gissane angles, and the articular orientation. Patients also had a CT scan performed. Patients were scheduled for surgical intervention within the first 1-4 days in order to ease manipulation of the fracture fragments. Like Thermann et al. [17], we believe that simple intra-articular fractures with minor displacement of the posterior facet articulation may be reduced by using small portals and a percutaneous technique.

Small wire circular frames were used on all patients. The patient was placed supine on an operating table with a radiolucent top. A tourniquet was usually applied but rarely inflated. The frames used consisted of one tibial ring attached to the mid-tibia, with one wire fixing the tibiofibular joint and the second fixing solely the tibia. The footplate was attached to the metatarsals, talus, and calcaneus.

The tibial ring was positioned to allow 1-2 fingerbreadths between the ring and the skin.

The first wire was directed at a $30^{\circ}$ angle in a medial to lateral direction, with care being taken to ensure purchase 
of both cortices of the tibia and fibula. The second wire was then passed from lateral to medial through the anterior tibia only. These wires were then tensioned to the manufacturer's specifications (Fig. 1).

The footplate was then attached to the forefoot via two crossed wires through the distal metatarsals, ensuring that as many cortices as possible had been crossed. These wires were subsequently tensioned. A wire was then passed through the talar neck and tensioned. A final wire was passed through the calcaneal fragment immediately inferior to the posterior facet before it was bent into the shape of an inverted "U," so that the posterior fragment was brought down into position when tensioned (Fig. 2).

Olive wires were used through the calcaneus to apply side-to-side compression through the fragments and to reduce heel widening. This fixation between the tibia and forefoot allowed manipulation and distraction forces to be focused on the calcaneal fractures rather than the ankle, midfoot, or ligamentous structures (Fig. 3) [12].

At this point, three bars were loosely connected to the tibial ring and footplate, with care being taken to position the bars in multiple planes to better resist shifting of the fracture once reduced. The width of the calcaneus was restored by manual pressure on either side of the calcaneus, while distal traction was applied by pulling the calcaneus distally and posteriorly. In order to aid reduction of the posterior facet, percutaneous manipulation with a Steinmann or Schanz pin in the tuberosity was used like a joystick in tongue-type fractures.

For joint depression types, we used the approach described by Gallie in 1943 [5]. An incision two and onehalf inches long was made along the lateral side of the achilles, down to the os calcis. This was deepened through the superficial and deep fascia, through the transverse intermuscular septum, which separates the soleus and gastrocnemius from the flexors of the toes, and through the

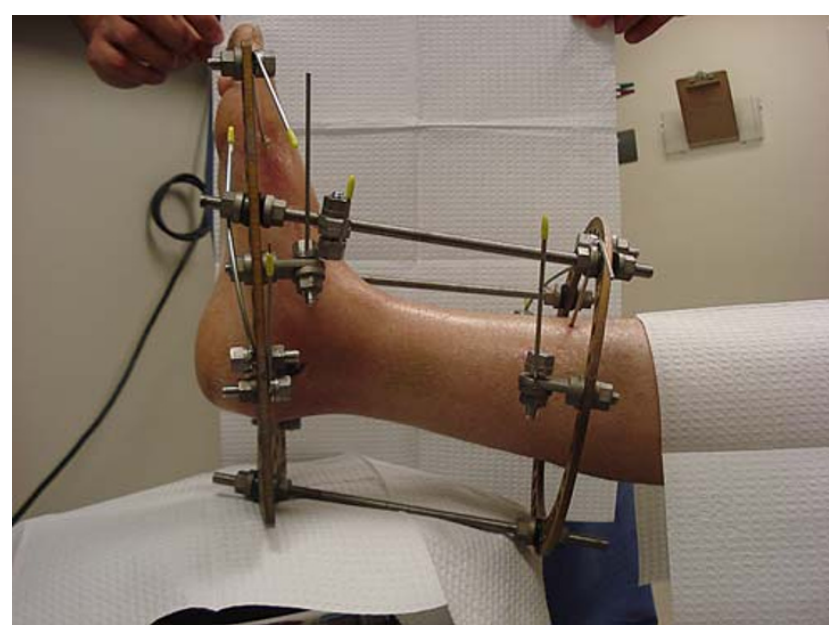

Fig. 1 LIMA frame on right leg

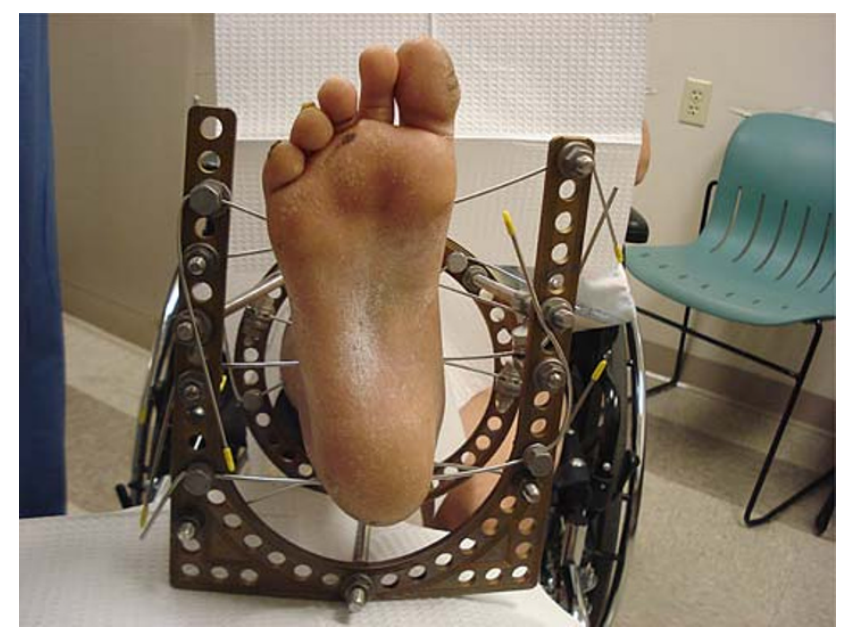

Fig. 2 LIMA frame

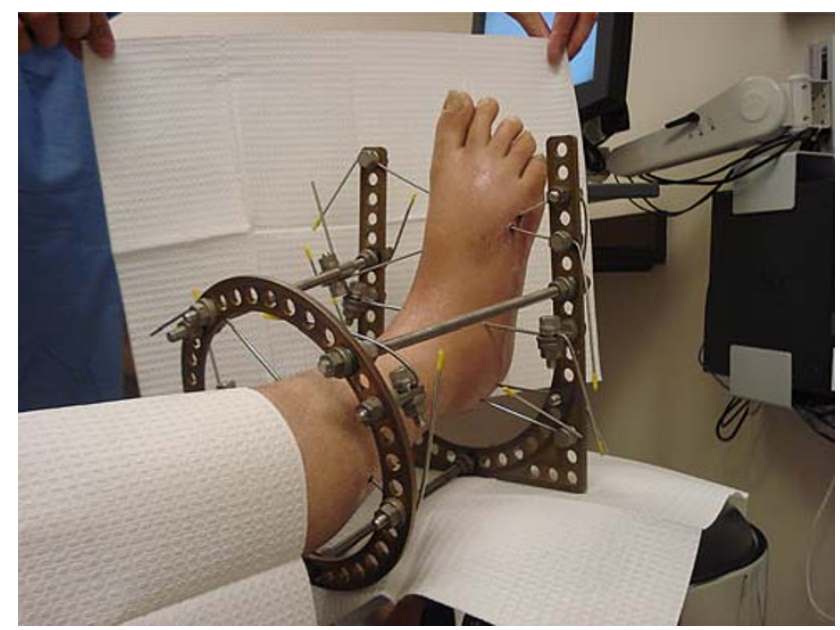

Fig. 3 LIMA frame

fat that fills the space between the posterior end of the subtalar joint and the sheath of the tendoachilles.

With the help of a retractor, the posterior aspect of the lower end of the tibia, the belly of the flexor hallucis longus, the ligaments of the ankle joint and the subtalar joint were then brought into view, and the position of the subtalar joint was demonstrated by rocking the calcaneus sideways. The articular surface was then elevated under direct vision using a small periosteal elevator.

Once adequate reduction was achieved, the bars connecting the tibial ring and footplate were locked into place.

The wounds were closed with mattress sutures to avoid tension on the edges. These sutures were removed two weeks postoperatively. Pin care instruction (using normal saline solution three times a day) and a non-weight-bearing status were given to all patients until removal of the frame at around 2-3 months. The timing in frame was guided by clinical and radiological healing parameters. This was 
followed by the application of a bellow knee soft cast application for four weeks, followed by physical therapy for ankle and foot range or motion, weight-bearing and gait exercises.

\section{Results}

\section{Complications}

We had no secondary reconstruction procedures. A total of five complications were noted, including three superficial infections treated successfully with oral antibiotics, one skin ulcer around the heel, and one deep infection which required removal of the frame and the application of a soft cast 28 days after the application of the fine wire circular frame. The patient's injury was an open calcaneal fracture secondary to a gunshot wound. The patient smoked two packets of cigarettes per day.

\section{Radiological outcome}

The Böhler's angle, Gissane's angle and posterior subtalar joint space averaged, respectively, $16^{\circ}(5-30), 115^{\circ}$ (60-145), and $2.9 \mathrm{~mm}(0-5.9)$ preoperatively whilst the average measurements at the last follow-up appointment $\mathrm{X}$-ray were, respectively, $17^{\circ}(0-33), 106^{\circ}(75-130)$, and $2 \mathrm{~mm}(0-5.9)$.

The difference between the preoperative values and the follow-up measurements was not statistically significant using Student's $t$-test $(P=0.8 \quad[\mathrm{CI} 95 \%-3-2.5]$ for
Böhler, $P=0.2$ [CI 95\% -6.8-8.3] for Gissane, and $P=0.4$ [CI 95\% $-0.26-2.45$ ] for the posterior subtalar joint space).

Functional outcome with the AAOS foot and ankle score

We assessed patient functional status using the American Academy of Orthopaedic Surgeons foot and ankle score. This score is validated [10], and normative values for the general population have been studied [8]. The score is a patient-derived questionnaire consisting of 25 questions on ankle stiffness, swelling, pain when walking on uneven grounds, when walking on flat surfaces, when walking up or down stairs, pain when lying in bed, history of ankle giving way during various activities, balance, ability to get around, pain when putting on socks, pain when standing, and ability to cope with activities of daily living and with work. Results are presented in Table 1.

We contacted 15 of 16 patients a minimum of twelve months after their injury (mean 25, range 12-48). We were unable to contact one patient.

The standardized scores ranged from 42 to 96 , with a mean of 80 and a standard deviation of 19. In order to provide the user with a method of interpreting/comparing the results for a given patient's functioning to a healthy population, normative values were created.

To make the scores comparative across various scales, the Normative Data Study's results were transformed for each scale so that each has a mean normative score of 50 . Thus, a patient scoring above 50 on a particular scale is
Table 1 AAOS scores of 15 of our 16 patients with corresponding Essex Lopresti classification, skin status and angles

\begin{tabular}{lllllll}
\hline Patients & $\begin{array}{l}\text { Foot and ankle } \\
\text { core scale: } \\
\text { standardized }\end{array}$ & $\begin{array}{l}\text { Foot and ankle } \\
\text { core scale: } \\
\text { normative }\end{array}$ & $\begin{array}{l}\text { Essex } \\
\text { Lopresti }\end{array}$ & $\begin{array}{l}\text { Skin } \\
\text { status }\end{array}$ & $\begin{array}{l}\text { Böhler } \\
\text { angle }\end{array}$ & $\begin{array}{l}\text { Gissane } \\
\text { angle }\end{array}$ \\
\hline 1 & 84 & 43 & Joint depression & Closed & 20 & 135 \\
2 & 85 & 43 & Tongue & Closed & 25 & 120 \\
3 & 92 & 49 & Tongue & Closed & 20 & 133 \\
4 & 80 & 39 & Joint depression & Closed & 18 & 124 \\
5 & 94 & 50 & Joint depression & Open & 10 & 145 \\
6 & 96 & 52 & Joint depression & Closed & 15 & 140 \\
7 & 93 & 50 & Joint depression & Closed & 17 & 116 \\
8 & 57 & 21 & Joint depression & Closed & 17 & 100 \\
9 & 51 & 16 & Joint depression & Closed & 14 & 127 \\
10 & 56 & 20 & Tongue & Closed & 12 & 120 \\
11 & 92 & 49 & Tongue & Closed & 25 & 105 \\
12 & 42 & 8 & Joint depression & Closed & 5 & 95 \\
13 & 93 & 50 & Joint depression & Closed & 18 & 60 \\
14 & 93 & 50 & Tongue & Closed & 30 & 115 \\
15 & 93 & 50 & Joint depression & Open & 15 & 100 \\
Means & 80 & 40 & & & 16.28 & 115.35 \\
\hline
\end{tabular}


above the general population's average, while a patient scoring below 50 on a scale is below the general, healthy population's norm.

A mean for the overall scale scores was derived from the general United States population and is set at 50, with a standard deviation of ten [20]. We also looked at the rate of patients who had returned to their preinjury work. Out of 15 patients, 12 were working before their injuries. Ten patients of these 12 patients are back at work.

\section{Discussion}

In our study, we looked at a consecutive series of sixteen patients with displaced intra-articular calcaneal fractures treated with fine wire circular frame. Although there is no clear evidence that allows us to state what the best management for this type of fractures is, it seems that nonoperative treatment of displaced intra-articular fractures is not appropriate. In fact, several authors have compared nonoperative management of these fractures to ORIF with similar conclusions.

Thodarson and Krieger [18] rated their results for 28 patients (17 operative and 11 nonoperative) using the AOFAS. This study reported statistically significant superior results in the operative group compared with the nonoperative group. The operative group had an increase of Böhler's angle from $11^{\circ}$ to $26^{\circ}$, and the average residual displacement of the posterior facet was $4.7 \mathrm{~mm}$ in the nonoperative group compared with $1.1 \mathrm{~mm}$ in the operative group. Only $25 \%$ of the operatively treated patients reported having pain with motion, compared with $100 \%$ in the nonoperative group.

There are many hypotheses to explain the poor results for nonoperative management; the most widely accepted concept is that patients with anatomically reduced fractures will have a better function then patients with unreduced fracture and articular surface (although this depends on the energy transfer to the joint during the injury). Sanders et al. [15] conclude that an anatomical reduction of the articular surface is necessary to obtain good to excellent functional outcome, but does not guarantee it (most likely due to the damaged articular surface during the impact). Furthermore, they show a clear correlation between the fracture comminution, the adequacy of reduction, and patients' outcome, showing that an anatomic reduction was achieved in $86 \%$ of cases of two-part fractures, which resulted in $73 \%$ good and excellent outcomes. In three-part fractures, $60 \%$ of patients had an anatomic reduction, with $70 \%$ of patients rated as good outcomes. Good and excellent results were only achieved in those cases where an anatomic reduction was obtained.
Similarly, Letournel [11] followed up 99 cases of calcaneal fractures at an average of two years post-ORIF. One-third of the cases rated fair, with none of these patients having a good initial fracture reduction, one-third rated good, and the final third rated excellent. The author also reported a $10 \%$ failure rate (classified as bad), with six technical failures and three infections.

Loucks et al. [13] looked at 95 fractures in 88 patients. Radiographic results were measurements of Böhler's angle on plain X-rays. They used the visual analog scale for pain and SF-36 as a measurement of the functional outcome of patients. This prospective randomized cohort study indicated that patients initially presenting with a severely depressed Böhler's angle had a poor two-year outcome regardless of treatment. Also, fractures of lesser initial displacement, as measured by Böhler's angle, had higher functional scores on both VAS and SF-36 scoring scales.

In contrast, our radiological outcome, looking at Böhler's angle, Gissane's angle and the posterior subtalar joint space at the last follow-up appointment, did not show any significant improvement when compared to the preoperative status; in fact, the difference between preoperative Böhler's angle, Gissane's angle and posterior subtalar joint space and their respective postoperative values was not statistically significant.

There is a degree of interobserver and intraobserver variability in the measurement of angles on X-ray, which needs to be taken into consideration. If these radiological measurements can reflect the accuracy of the reduction, then we have found no correlation between the accuracy of reduction and functional outcome. We could not find a correlation between patients with good correction of Böhler's angle and high functional score either. These findings seem to go against the current philosophy of restoring radiological landmarks to obtain good function. We realize that, without values for outcome measures such as range of movement of the ankle and the subtalar joint, our results are incomplete. Furthermore, the small number of patients in this paper is a recognized weakness of our study. On the other hand, we feel that the most important outcome measures are the patient-derived measures of satisfaction and function; more so than clinician-derived subjective values.

The advantage of ORIF is the reduction of the subtalar and calcaneocuboid joints, as well as the restoration of calcaneal height, length, translation and varus/valgus alignment. This is usually achieved through a lateral approach, which has the advantage of excellent exposure to the posterior facet, calcaneocuboid joint, lateral wall and the tuberosity. However, complications associated with this approach involve up to one in four patients [7], including deep infection, skin necrosis, peroneal tendonitis and screw penetration into the subtalar articular surface. 
Several authors have consequently used indirect reduction and application of fine wire circular frames to treat intra-articular calcaneal fractures with poor skin condition. Emara et al. [2] treated 12 patients with Sanders type III fractures and poor skin condition. The technique used was an open reduction of the articular surface of the subtalar and calcaneocuboid joints. Using a fine wire fixator and distraction completed the reduction. A measure of the function of patients was achieved using the AOFAS and compared to the function of 20 patients with Sanders type III fracture and adequate skin condition who had been treated with ORIF. At a mean of 20 months, both groups had similar functional and radiographic outcomes. The ORIF group had higher postoperative complications, with peroneal tendinosis, hardware penetration into the subtalar joint, superficial wound infection, and superficial sensory nerve injury that subsided ten months after the surgery.

McGarvey et al. [14] followed up a series of 31 patients with 33 Sanders II, III or IV calcaneal fractures treated with indirect reduction (through a percutaneous plantar skin incision) and external fixation (using small wire circular frame external fixation) at a minimum of six months (six months to four years). The average AOFAS score for the 18 available patients was 66 (42-92), increasing to 74 for patients with at least ten months of follow-up and to 77 for patients with an isolated calcaneal fracture. They had no secondary reconstructive procedures, and all open fractures healed and maintained soft tissue coverage. There were 11 complications, including nine superficial pin track infections that responded well to oral antibiotics, one deep infection in a diabetic smoker who presented ten days postinjury, and one patient with a small area of skin necrosis.

Our results compare favorably to these findings, with no secondary reconstruction procedures. One patient underwent a second operation 28 days following the application of the ring fixator. This patient had an open fracture from a gunshot wound and was a two-pack-per-day cigarette smoker. His fixator was removed and the wound was irrigated. Following a course of antibiotics, the patient was reviewed in the clinic with a healed wound and no signs of infection. Three patients had superficial infection, which resolved with oral antibiotics and pin site care. Finally, one patient had a small ulcer on the heel that took two months to granulate and settle.

We used the AA FAS [10] as a functional outcome measure. We could not find any study looking at calcaneal fractures that used this scoring system, although it is validated and reliable. The standardized scores ranged from 42 to 96 , with a mean of 80 and a standard deviation of 19 . The mean of a healthy population is 93.2 with a standard deviation of 12.3. Thodarson et al. [19] recently used the AAOS foot and ankle score, looking at women undergoing corrective surgery for hallux valgus (scoring was performed preoperatively). The scores ranged from 72 to 80 .

In our series of patients, four patients showed standardized mean scores of below $60(42,56,51$, and 57). Without these poor functional scores, our mean standardized score would have been 89.25. When compared to the mean of a healthy population with a $t$-test, the difference was not found to be significant, with $P=0.35$ and a $95 \%$ confidence interval for this difference from -12.549 to 4.549.

Among these patients were three males and one female with an average age of 49 years. These four patients had closed injuries and were otherwise healthy. Only one was a smoker. The four injuries were joint-depression types. Three patients had no other associated injuries, while one patient had other fractures of the foot. There was no particular delay in the operation, with a mean time from injury to surgery of 12 days. Two of these four patients had a superficial infection that was suppressed with antibiotics. Surprisingly, when grouped together, these patients had a mean change in Böhler's angle from injury to the reduction of $+7^{\circ}$, which should be compared to the mean of the 16 patients, $+0.6^{\circ}$. We would expect that this increase in Böhler's angle would lead to a better outcome, but it had the opposite effect in our series. There was no difference between this small group of patients with poor outcome and the rest of the group when other radiological measurements were considered (Gissane angles and posterior subtalar joint space).

The best management for intra-articular calcaneal fractures is still controversial. There is evidence for the idea that displaced fractures should be treated surgically rather then nonoperatively. In fact, the functional outcome of patients treated without surgical intervention is poor when compared to the outcome of patients treated with an ORIF procedure.

Unfortunately, ORIF procedures are not always possible, especially when skin quality is poor due to excessive swelling or blisters, or when the fracture is too comminuted. Furthermore, the complication rate of ORIF is as high as $25 \%$ in most surgeons' hands. Several authors have used a fine wire circular frame to treat displaced intraarticular calcaneal fractures.

We treated 16 consecutive patients over a period of three years with this technique. Our results, as presented above, show no secondary reconstruction procedures, one deep infection, three superficial infections and one skin ulcer, all suppressed within three months of fracture fixation. Our radiological outcome, evaluated by examining Böhler's angle, Gissane's angle and the posterior subtalar joint space at the last follow-up appointment, did not show any significant improvement when compared to the preoperative status, but functional outcome score based on the 
AAOS foot and ankle score was good. Ten of the 12 working patients are now back performing their normal work. Stable fixation with a low complication rate and good function can be achieved by using a minimal approach to the subtalar joint combined with a fine wire circular frame and a deformity correction technique to reduce the fracture. This technique can be used in almost every patient with an intra-articular calcaneal fracture, especially if the skin condition is not adequate for an ORIF.

Conflict of interest statement The authors declare that they have no conflict of interest related to the publication of this manuscript.

\section{References}

1. Böhler L (1931) Diagnosis, pathology, and treatment of fractures of the os calcis. J Bone Joint Surg 13:75-89

2. Emara KM, Allam MF (2005) Management of calcaneal fractures using the Ilizarov technique. Clin Orthop Relat Res 439:215-220

3. Essex-Lopresti P (1952) The mechanism, reduction, technique, and results in fractures of the os calcis. Br J Surg 39:395-419

4. Folk JW, Starr AJ, Early JS (1999) Early wound complications of operative treatment of calcaneum fractures: analysis of 190 fractures. J Orthop Trauma 13:369-372

5. Gallie WE (1943) Subastragalat arthrodesis in fractures of the os calcis. J Bone Joint Surg 43:731-736

6. Gissane W (1947) Discussion on "Fractures of the os calcis" (Proceedings of the British Orthopaedic Association). J Bone Joint Surg 29:254-255

7. Harvey EJ, Grujic L (2001) Morbidity associated with ORIF of intra-articular calcaneal fractures using lateral approach. Foot Ankle Int 22:868-873

8. Hunsaker FG et al (2002) The American Academy of Orthopaedic Surgeons outcomes instruments: normative values from the general population. J Bone Joint Surg 84:208-215
9. Ibrahim A, Beiri M, Azzabi A, Best G, Taylor D (2003) Reliability and validity of the subjective component of the American orthopaedic foot and ankle society clinical rating scales. J Foot Ankle Surg 46(2):65-74

10. Johanson NA, Liang MH, Daltroy L, Rudicel S, Richmond J (2004) American academy of orthopaedic surgeons lower limb outcomes assessment instruments. reliabilty, validity, and sensitivity to change. J Bone Joint Surg 86:902-909

11. Letournel E (1993) Open treatment of acute calcaneal fractures. Clin Orthop 290:60-67

12. Levin LS, Nunley JA (1993) The management of soft tissue problems associated with calcaneal fractures. Clin Orthop 290:151-156

13. Loucks C et al (1999) Bohler's angle: correlation with outcome in displaced intra-articular calcaneal fractures. J Ortho Trauma 13:554-558

14. McGarvey W et al (2006) Calcaneal fractures: indirect reducation and external fixation. Foot Ankle Int 27:494-499

15. Sanders $\mathrm{R}$ et al (1993) Operative treatment in 120 displaced intraarticular calcaneal fractures. Results using a prognostic computer tomography scan classification. Clin Orthop 290:87-95

16. Sangeorzan BJ, Benirschke SK, Car JB (1995) Surgical management of the os calcis. Instr Course Lect 44:359-370

17. Thermann H, Krettek C, Hufner T (1998) Management of calcaneal factures in adults: conservative versus operative treatment. Clin Orthop 353:107-124

18. Thodarson DB, Krieger LE (1996) Operative versus non-operative treatment of intra-articular fractures of the calcaneus: a prospective randomized trial. Foot Ankle Int 17:2-9

19. Thodarson DB et al (2005) Age adjusted baseline data for women with hallux valgus undergoing corrective surgery. J Bone Joint Surg 87:66-75

20. AAOS (2008) Outcomes instruments and information (webpage). http://www.aaos.org/research/outcomes/outcomes_documentation. asp, Last Accessed 25 Nov 2008 